\title{
Where to begin? Defining national strategies for implementing the 2030 Agenda: the case of Switzerland
}

\author{
Thomas Breu $^{1,4} \oplus \cdot$ Michael Bergöö $^{2,3} \cdot$ Laura Ebneter $^{1} \cdot$ Myriam Pham-Truffert $^{1,2} \cdot$ Sabin Bieri ${ }^{1} \cdot$ Peter Messerli ${ }^{1,4}$. \\ Cordula Ott ${ }^{1} \cdot$ Christoph Bader $^{1}$
}

Received: 6 December 2019 / Accepted: 11 August 2020 / Published online: 26 August 2020

(C) The Author(s) 2020

\begin{abstract}
Five years after adoption of the 2030 Agenda, there is a general lack of progress in reaching its Sustainable Development Goals - be it on national, regional, or global scales. Scientists attribute this above all to insufficient understanding and addressing of interactions between goals and targets. This study aims to contribute to the methodological conceptualization of the 2030 Agenda's implementation at the national level. To this end, taking the case of Switzerland, we tested and enhanced existing approaches for assessing interactions among the 2030 Agenda's targets and for analysing the systemic relevance of priority targets. Building on our insights, the article concludes with an eight-step proposal for creating knowledge to support national 2030 Agendas.
\end{abstract}

Keywords Sustainable development $\cdot$ National 2030 Agenda strategies $\cdot$ SDG interactions $\cdot$ Indicators $\cdot$ Monitoring and evaluation $\cdot$ Switzerland

Handled by Siddharth Sareen, Postdoctoral research fellow University of Bergen, Norway.

Electronic supplementary material The online version of this article (https://doi.org/10.1007/s11625-020-00856-0) contains supplementary material, which is available to authorized users.

Thomas Breu

thomas.breu@cde.unibe.ch

Michael Bergöö

bergoo.michael@gmail.com

Laura Ebneter

laura.ebneter@cde.unibe.ch

Myriam Pham-Truffert

myriam.pham-truffert@cde.unibe.ch

Sabin Bieri

sabin.bieri@cde.unibe.ch

Peter Messerli

peter.messerli@cde.unibe.ch

Cordula Ott

cordula.ott@cde.unibe.ch

\section{Introduction}

In 2015, the United Nations (UN) General Assembly adopted Resolution 70/1, Transforming our world: The 2030 Agenda for Sustainable Development, with its 169 targets clustered into 17 Sustainable Development Goals (SDGs) (United Nations 2015). Unlike the preceding Millennium Development Goals, the 2030 Agenda was equipped with a comprehensive follow-up and review process. To monitor progress on its implementation, the UN adopted a global indicator

Christoph Bader

christoph.bader@cde.unibe.ch

1 Centre for Development and Environment, University of Bern, Mittelstrasse 43, 3012 Bern, Switzerland

2 Sustainable Development Solutions Network Switzerland, Heinrichstrasse 147, 8005 Zurich, Switzerland

3 Biovision Foundation for Ecological Development, Heinrichstrasse 147, 8005 Zurich, Switzerland

4 Institute of Geography, University of Bern, Hallerstrasse 12, 3012 Bern, Switzerland 
framework that currently comprises 231 unique indicators. This framework forms the backbone of annual SDG progress reports prepared by the Secretary-General. Because the Agenda's implementation largely relies on national progress, most signatory states have set up national implementation plans, often coupled with corresponding monitoring and indicator systems. In many UN member states, these instruments form the basis for regular monitoring of progress and its documentation in voluntary national reviews (VNRs) submitted to the High-Level Political Forum on Sustainable Development (HLPF).

Four years after the adoption of the 2030 Agenda, the Global Sustainable Development Report 2019 revealed a general lack of progress in reaching the SDGs-whether on national, regional, or global scales (Independent Group of Scientists 2019). Scientists attribute this above all to insufficient understanding and addressing of interactions between goals and targets. They underline that implementation of the 2030 Agenda requires a systemic approach focusing on interlinkages between goals and targets (Griggs et al. 2014; Nilsson et al. 2016; Messerli et al. 2019 and others). The challenge lies in identifying and countering inherent conflicts (trade-offs), while harnessing and building on potential synergies (co-benefits) between the 169 targets. Accordingly, the focus must be on identifying and harnessing positive interactions, where targets mutually reinforce each other in a desired way, while limiting negative interactions, where targets undermine or contradict each other (Le Blanc 2015; ICSU 2017; Stafford-Smith et al. 2017, and others). Meanwhile, policymakers, civil society organizations, and the private sector are increasingly coming to share the view that meaningful global and national priority-setting is only possible if we disentangle and tackle synergies and trade-offs among targets.

Given the 2030 Agenda's complexity, it must-despite its being indivisible - be split up into tangible entities in analytical practice. This, however, can cause attention to be focused on targets that are relatively easy to achieve; which in turn poses the risk that sectoral approaches and siloed policies-which the 2030 Agenda was intended to overcomemight continue to prevail (Pradhan et al. 2017; Breuer et al. 2019). A UN synthesis study of all 43 VNR reports available in 2017 confirms these fears (United Nations 2018). It shows that 23 reports refer only loosely to trade-offs or similar terms, and as few as 7 mention specific trade-offs. Against this background, the question of how to address interactions among goals and targets has recently found its way into UN guidelines and handbooks designed to support countries in their ongoing reporting exercises (UN-DESA 2019). One example is the Rapid Integrated Assessment (RIA) tool, which provides steps and templates to help policymakers determine the relevance of the various SDGs and their interlinkages in a country context (UNDP 2017). Even though the number of concepts, frameworks, and approaches addressing SDG interlinkages has increased, implementation of such a systemic approach is seriously constrained by a lack of in-depth understanding of how targets affect each other (see Tosun and Leininger 2017; Breuer et al. 2019). From a practical point of view, assuming a systemic perspective on interlinkages would imply dealing with 28,392 potential first-order target interactions (i.e., 169 times 169, minus 169 self-loops). A full systemic analysis is thus only feasible if it is done quantitatively - but the limited availability and adequacy of data seriously hamper such endeavours. In view of this situation, major efforts are being made to further develop global and national indicator systems (International Council for Science 2017; Pradhan et al. 2017; Zhou and Moinuddin 2017; Nilsson et al. 2018; Weitz et al. 2018) and to establish SDG governance support platforms like the Sustainable Governance Indicator Network (SGI) and others. Further limitations of data-driven approaches using correlation analysis (see Pradhan et al. 2017; Zhou and Moinuddin 2017) relate to the fact that while they show first-order associations between targets, they provide no information about causalities (Breuer et al. 2019). Qualitative crossimpact matrix approaches (for an overview see Tosun and Leininger 2017; Breuer et al. 2019), which do not depend on statistical data (Nilsson 2016), are likewise confronted with various limitations. Most notably, expert judgments of interactions are, by definition, subjective. Accordingly, resulting selections of targets to be considered can only give rough indications for priority-setting in national sustainable development strategies.

A key aspect to be considered in strategy implementation is policy coherence. This is reflected most notably, but not exclusively, in the 2030 Agenda's target 17.14, to "Enhance policy coherence for sustainable development" (PCSD). The past years have seen the development of a growing number of PCSD assessment and monitoring frameworks (OECD 2016; Collste et al. 2017; Nilsson and Weitz 2019). The basic idea behind PCSD is to avoid, nationally and internationally, contradictions (i.e., tradeoffs) between individual policies, while fostering synergies among them on behalf of the SDGs (Dohlman 2016; Zeigermann 2018). Establishing PCSD involves balancing diverging interests and accounting for winners and losers in society. In line with the 2030 Agenda's key normative principle of leaving no one behind (LNOB), PCSD requires that policy decisions be based on a transparent consultation and negotiation process that reflects the interests and perceptions of different stakeholder groups. Meanwhile, the interests of less powerful groups such as future generations, low-income countries, and those demanding the protection of global public goods (e.g., the environment) are often neglected or sidelined in practice (Breu et al. 2017). 
Switzerland, like many other countries, is seeking ways of meeting its national commitment to the 2030 Agenda. Stimulated by the UN Conference on Environment and Development (Rio de Janeiro 1992) and concerned about growing social and environmental concerns, in 1999, the Swiss people voted to include sustainable development in the national constitution as one of the purposes of the Swiss Confederation. However, no federal legislation has been enacted so far to detail the obligations and modalities for achieving this vision. Instead, implementation of the constitutional obligation to foster sustainable development is guided by means of national sustainable development strategies $(1997,2002$, 2008, 2012, 2016). At the international level, the Swiss government was prominently involved in the process of defining and reaching agreement on the 2030 Agenda (Swiss Civil Society Platform Agenda 2030). After Switzerland's ratification of the 2030 Agenda, the federal government established an institutional mechanism for its implementation. The framework for implementation is provided by the Swiss Sustainable Development Strategy 2016-2019 (Swiss Federal Council 2016), while a national 2030 Agenda Steering Committee-composed of senior management-level representatives of key federal agencies and supported by an advisory group of representatives from Switzerland's private sector, NGOs, and scientific community-is instrumental in guiding the strategy and review process.

Switzerland is currently developing its Sustainable Development Strategy 2020-2030, which will define the government's political priorities. This reference framework, along with an action plan for the period of 2020-2023, aims to ensure PCSD in Switzerland. To date, the country has but a weak statutory framework to guide decision-making for sustainable development and related consultations and hearings, such that balancing of interests remains piecemeal (Breu et al. 2017). The upcoming sustainable development strategy will be inspired by the concept of transformative pathways proposed in the UN Global Sustainable Development Report 2019 (Independent Group of Scientists 2019). This concept focuses on a small number of thematic entry points as well as levers that can be used across all entry points to achieve transformations.

Monitoring and evaluation of the current and future strategy as well as Switzerland's VNR reporting to the United Nations (Swiss Confederation 2018) relies, among other things, on the database prepared within the national sustainable development monitoring system MONET 2030. MONET (abbreviation for the German Monitoring der nachhaltigen Entwicklung, "Monitoring of Sustainable Development") is a joint activity of the Federal Statistical Office (FSO), the Federal Office for the Environment (FOEN), the Federal Office for Spatial Development (ARE), and the Swiss Agency for Development and Cooperation (SDC) that was partially expanded in 2017 in response to requirements of the 2030 Agenda (hence, "MONET 2030"). The system currently comprises 85 indicators for monitoring national implementation of the 2030 Agenda. According to the OECD (2018), the Swiss monitoring system integrates "the three dimensions of sustainable development, considers interactions between them as well as intergenerational and transboundary dimensions", although the 2018 country report also reveals specific limitations with regard to establishing PCSD (OECD 2018).

The present study aims to contribute to the methodological conceptualization of the 2030 Agenda's implementation at the national level. Using the case of Switzerland, we tested and enhanced existing approaches for assessing interactions among the Agenda's targets and for analyzing the systemic relevance of priority targets. Building on a statistical analysis of a stakeholder survey of sustainable development priorities, we assessed systemic relationships between targets following the qualitative approach by Nilsson et al. (2016). We then compared the results with those from a quantitative approach (Pradhan et al. 2017) using indicators from Switzerland's official sustainability monitoring system. The results of the qualitative assessment were further analyzed to unravel targets' potential to act as systemic buffers or multipliers in national implementation strategies and as concrete potential transformation pathways. Building on our insights, we conclude this article by proposing an 8-step process for the formulation and implementation of national sustainable development strategies.

\section{Data and methods}

This study is based on two sets of data. On the one hand, we used data derived from the results of a stakeholder consultation on the status of implementation of the 2030 Agenda in Switzerland carried out by the federal government in preparation of its VNR report (Swiss Confederation 2018). The information was gathered in an online consultation coordinated by the Federal Office for Spatial Development (ARE). A total of 167 representatives from non-governmental (160) and public-sector (7) organizations and institutions appraised Switzerland's targets for implementation of the 2030 Agenda. The responses collected in this stakeholder consultation provide an in-depth view of how Swiss organizations prioritize the various targets, where they see a need for action, and what expertise and experiences they can offer to support implementation of the 2030 Agenda in Switzerland. On the other hand, we analyzed data from the MONET 2030 sustainable development monitoring system, which uses 85 selected indicators to assess the status of implementation of the 2030 Agenda in Switzerland. We analyzed these two data sets in a three-step procedure. First, we defined criteria to identify priority targets based on 
stakeholders' assessments using an analytic hierarchy process (AHP). Second, we established a cross-impact matrix for Switzerland's priority targets, following the example of a study in Sweden (Weitz et al. 2018). On this basis, we performed an expert judgment as described by Nilsson et al. (2016). Third, we applied network analysis, including a prospective structural analysis, with three aims: to understand the systemic impact of targets, to establish an order of priority for policy action on the SDGs in Switzerland, and to suggest concrete transformation pathways and entry points for implementation using the identified synergies. Finally, we analyzed interactions between targets using the relevant data time series from MONET 2030 and calculating Spearman's rank correlation according to Pradhan et al. (2017) and Kroll et al. (2019).

\section{Selection of priority targets in Switzerland}

To determine priority targets for Switzerland as assessed by Swiss stakeholders, we clustered the 167 respondents of the stakeholder consultation into six stakeholder groups (Table 1). Then, out of five questions that the respondents had had to answer for each of the 169 SDG targets, we selected the two following questions, which are of direct relevance to this study:

- What do you think of the federal government's objectives? Are they going too far, are they insufficient, or do you agree with the proposed targets?

- What challenges must be addressed in order for Switzerland to reach its national-level targets and make an appropriate contribution internationally?

Following an analytic hierarchy process (AHP) - a method used to organize and analyze complex decisionswe decomposed the decision on priority targets into a hierarchy of more easily comprehensible sub-problems, each of which we could analyze independently (Fig. 1).

Table 1 Clustering of respondents into six stakeholder groups

\begin{tabular}{llc}
\hline Stakeholder group & $\begin{array}{l}\text { Number of } \\
\text { respondents }\end{array}$ & $\begin{array}{l}\text { Percentage of } \\
\text { respondents }\end{array}$ \\
\hline Civil-society organizations & 33 & 19.8 \\
Private-sector organizations & 41 & 24.6 \\
Environmental organizations & 22 & 13.2 \\
Social organizations & 46 & 27.5 \\
Scientific institutions & 18 & 10.8 \\
Public-sector organizations & 7 & 4.2 \\
All & 167 & 100.0 \\
\hline
\end{tabular}

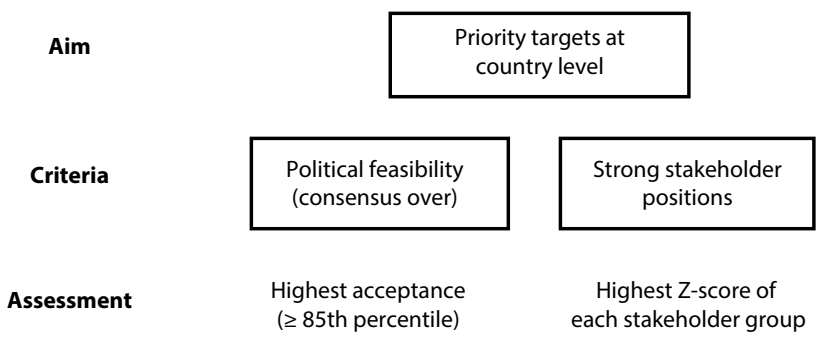

Fig. 1 Analytic hierarchy process to select priority targets for Switzerland

First, we defined the level of importance of each given target as the number of respondents having addressed it (see Appendix). Based on the criterion of political feasibility, we subdivided the level of importance into percentiles and defined all targets with a level of importance higher or equal to the 85 th percentile as priority targets (see Appendix). This resulted in a total of 27 priority targets. For the second criterion, strong stakeholder positions, we built $\mathrm{Z}$ scores $\left(z_{i}\right)$ for each stakeholder group within each target. $Z$ scores indicate by how much the acceptance rate of a target in a specific stakeholder group deviates from the overall mean acceptance rate of that target. Specifically, a Z score shows by how many standard deviations $(S)$ a specific score $\left(x_{i}\right)$ differs from the mean $(\bar{x})$.

$z_{i}=\frac{x_{i}-\bar{x}}{S}$.

We calculated the $\mathrm{Z}$ scores of all targets except the 27 already identified as priority targets and defined additional priority targets as those with the highest $\mathrm{Z}$ score in each stakeholder group. As there were six stakeholder groups, this resulted in six additional priority targets and an overall total of 33 priority targets.

\section{Assessment of target interactions in a cross-impact matrix}

We began investigating the systemic relationships between targets following the qualitative methodology proposed by Nilsson et al. (2016), which involves scoring relationships between priority targets on a 7-point scale. The scale distinguishes seven types of interaction, from canceling $(-3)$ to consistent $(0)$ to indivisible $(+3)$ (see Table 2$)$. In line with Nilsson et al. (2016) and Weitz et al. (2018) used this typology to score relationships between targets in a cross-impact matrix for the case of Sweden. While Weitz et al. (2018) chose the most important targets (two for each SDG) for Sweden based on expert judgement, we used the priority targets identified as described above on the basis of a broadbased stakeholder consultation. 
Table 2 Seven types of interactions between SDG targets. Source: Adapted from Weitz et al. (2018) and Nilsson et al. (2016)

\begin{tabular}{ll}
\hline Interaction label & Meaning \\
\hline +3 Indivisible & Progress on one target automatically delivers progress on the other \\
+2 Reinforcing & Progress on one target makes it easier to make progress on the other \\
+1 Enabling & Progress on one target creates conditions that enable progress on the other \\
\pm 0 Consistent & There is no significant link between the amount of progress made on the two targets \\
-1 Constraining & Progress on one target constrains options for delivering on the other \\
-2 Counteracting & Progress on one target makes it more difficult to make progress on the other \\
-3 Canceling & Progress on one target automatically reverses progress on the other
\end{tabular}

Following Nilsson et al. (2016), Weitz et al. (2018), and Gordon and Hayward (1968), we constructed a cross-impact matrix for the 33 selected targets. A cross-impact matrix is a tool to analyze the relationships between variables describing how the occurrence of the row variable would affect the column variable. Our matrix contains $1056(33 \times 32)$ interactions and is shown in "Results" (Fig. 2).

A group of sustainability experts did a qualitative evaluation of the interactions in the 1056 pairings of the matrix, drawing on their expert knowledge. To distribute the workload evenly, we divided the 1056 interactions according to the fields of expertise of five sustainability experts at the Centre for Development and Environment (CDE), after which each expert independently evaluated the interactions. Following this individual scoring exercise, the experts came together to discuss their scoring sheets. Questionable or disputed scores were discussed and jointly adjusted, resulting in an agreed final score for each of the relationships.

\section{Network analysis}

In our quest to further disentangle the systemic relationships between targets, we used network analysis to interpret the data resulting from the expert assessment of priority target interactions. Mostly building on the different methodologies developed in Weitz et al. (2018), Pham-Truffert et al. (2020) and Oliveira et al. (2019), we assessed the priority targets and their interactions as a directed and weighted network. We drew insights from the interactions (the edges of the network cells) to see how central a target (a node in the network) was in terms of its capacity to influence, or be influenced by, other targets. The expert assessment revealed how a given target A influences, positively or negatively, progress on a target $\mathrm{B}$. Using this information, we measured the differentiated influential relationships among targets by means of the following standard network measures:

- The weighted out-degree centrality is the sum of the weights of all edges going out from a given node. In our study, it corresponds to the given target's level of influence.
- The weighted in-degree centrality is the sum of the weights of all edges coming into a given node. In our case, it corresponds to the given target's level of dependence.

- The weighted degree centrality of a node in a directed network (a network in which the edges are directed) is the sum of that node's out-degree centrality and in-degree centrality.

\section{Identifying systemic buffers and multipliers}

Pham-Truffert et al. (2020) identified systemic buffers and multipliers of positive or negative effects by adapting concepts from Messerli (2000) to formal network analysis to gain insights from their database of SDG interactions (Pham-Truffert et al. 2020). Following the same approach, we handled the negative and positive values separately and used the weighted out- and in-degree centrality measures to identify the targets' potentially twofold role as systemic buffers and multipliers. We defined the positive and negative activity ratio of a given target in the network as its weighted out-degree centrality divided by its in-degree centrality. Furthermore, we defined its positive and negative interconnectedness as the weighted out-degree centrality times the weighted in-degree centrality. We then used these two calculations to place the positive and negative elements in a coordinate system, in which the logarithmic $x$-axis indicates the activity ratio and the $y$-axis indicates the interconnectedness (Pham-Truffert et al. 2020). Finally, we displayed the network according to our interpretation of the scatter plot-that is, highlighting systemic buffers as well as multipliers of both positive and of negative effects. This enabled us to identify: (1) the systemic buffers of positive effects; (2) the systemic buffers of negative effects; (3) the systemic multipliers of positive effects; and (4) the multipliers of negative effects. It is important to note that, according to this approach, a target can act as both a buffer and/or a multiplier on each positive or negative influence. Furthermore, the approach reveals how interconnected a given target is through these positive and negative influences.

Based on the study by Pham-Truffert et al. (2020), we further identified potential "virtuous cycles" that can serve 


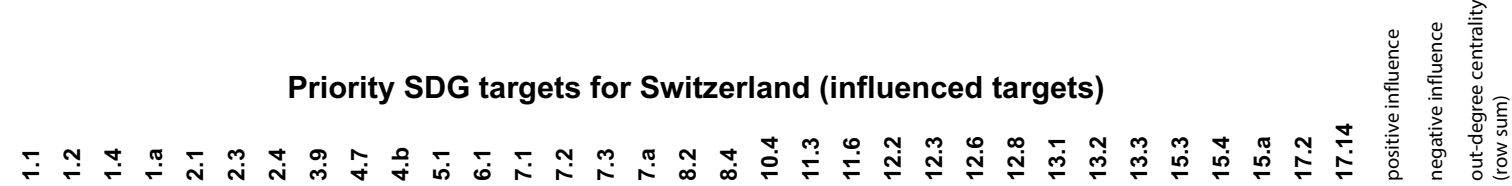

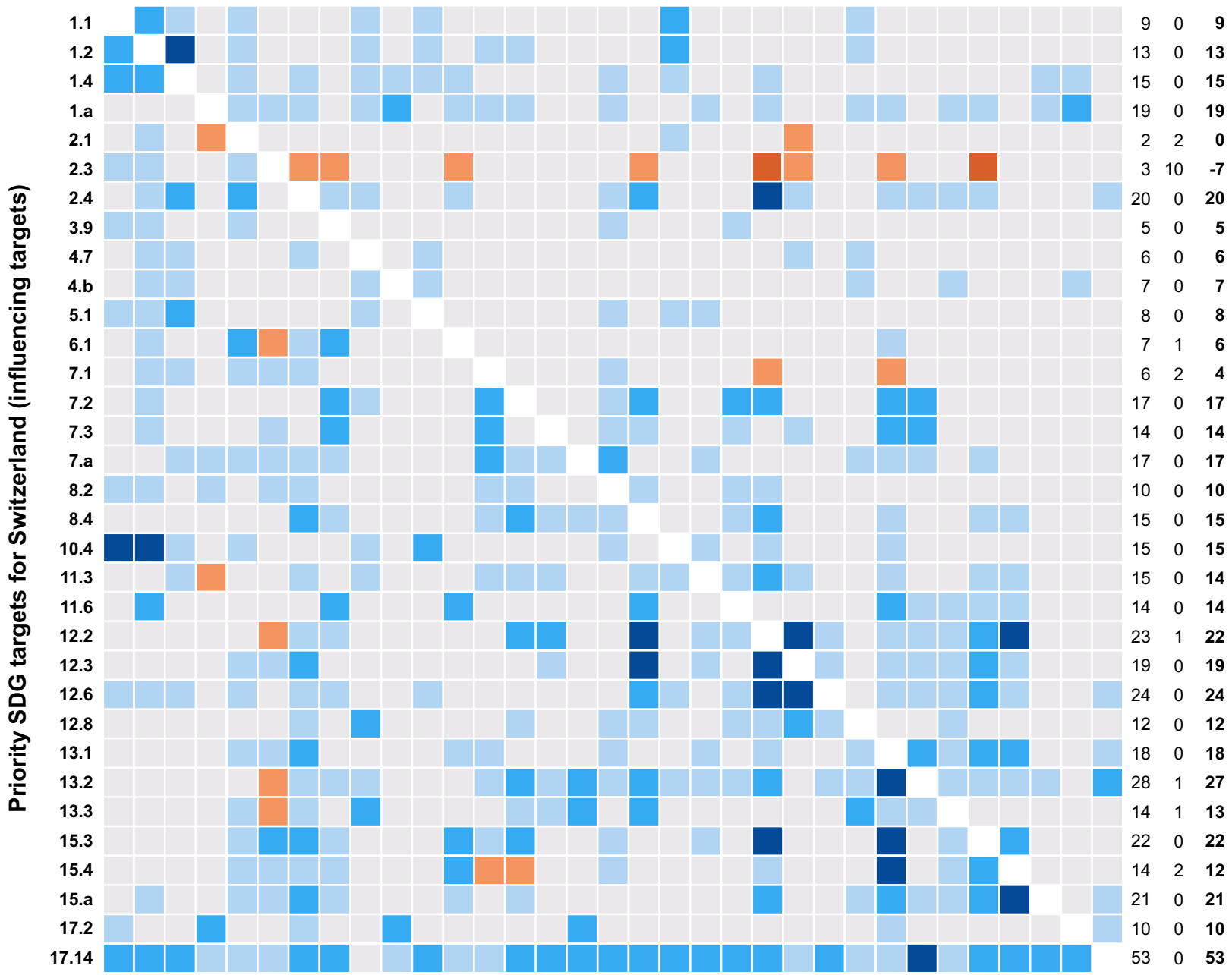

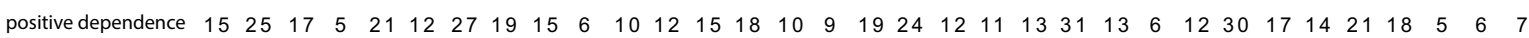

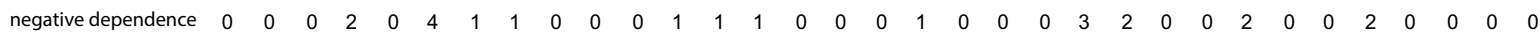

$\begin{array}{lllllllllllllllllllllllllllllllllll}\text { in-degree centrality } & 15 & 25 & 17 & 3 & 21 & 8 & 26 & 18 & 15 & 6 & 10 & 11 & 14 & 17 & 10 & 9 & 19 & 23 & 12 & 11 & 13 & 28 & 11 & 6 & 12 & 28 & 17 & 14 & 19 & 18 & 5 & 6 & 7\end{array}$

(column sum)

Fig. 2 Cross-impact matrix of 33 targets and their interactions in Switzerland. Interactions range from-2/counteracting (dark red) to +3 /indivisible (dark blue). The net influence of a target on all other

as concrete entry points for implementing the Swiss priority SDG targets. To this end, we extracted from all the strongest positive interactions (weighting 2 or 3 ) a list of all reinforcing positive directed loops of three targets (where target $\mathrm{A}$ influences $\operatorname{target} \mathrm{B}$, which influences target $\mathrm{C}$, which closes the loop by influencing target $\mathrm{A}$ ). We then condensed this list visually and ordered the cycles according to their topical closeness to the six entry points for transformation proposed in the Global Sustainable Development Report (GSDR) 2019. targets is shown by the row sum, while the column sum shows its net dependence on all other targets

\section{Prospective structural analysis}

To guide priority-setting in terms of where to focus action, we applied prospective structural analysis (PSA) to describe the system of interlinkages between Swiss priority targets and to allot specific roles to these targets. Following the method presented by Godet $(1991,2007)$ and its application by Oliveira et al. (2019), in a first step, we used the weighted out-degree centrality (row sum of cross-impact matrix) and the weighted in-degree centrality (column sum 
of cross-impact matrix) to build an influence-dependence matrix. Positioning all priority targets in such a matrix can indicate their role in implementing the 2030 Agenda at the national level. As proposed by Godet (1991), these roles are: (1) determinant targets-targets that are highly influential and little dependent, and thus constitute explanatory variables that condition the rest of the system; (2) relay targetstargets that are both highly influential and highly dependent, so that any action on them will affect other targets, which in turn will have feedback effects on the relay targets and might thus amplify or reduce the impact of the initial action; (3) resultant targets - targets with a small influence that are highly dependent on progress in other targets, such that achievement of these targets is largely influenced by determinant targets and relay targets; (4) regulator targets-targets with average influence and dependence, about which nothing can be said a priori; and (5) autonomous targetstargets that are little influential and little dependent (near to zero) and therefore less connected to the system, and can, thus, be viewed as having only marginal effects on the future system. Based on the results from the cross-impact matrix, we added a sixth function, (6) critical targets-targets that have considerable negative influence and are little dependent. Policies concerning these targets require special attention, since actions towards them can have detrimental effects on other targets. In a second step, we used the results from the above-described analysis of systemic buffers and multipliers to cross-check the roles assigned to the targets in the influence-dependence matrix.

\section{Quantitative analysis of target interactions}

To test the suitability of a quantitative analysis of interactions between priority targets we used the MONET 2030 data and checked the relationships using the nonparametric Spearman's rank correlation-the method applied in the global study by Pradhan et al. (2017) and Kroll et al. (2019). Spearman's rank correlation coefficient is "a measure of the strength of the association between two variables" (Hauke and Kossowski 2011) and, therefore, identifies synergies and trade-offs in statistical terms (Pradhan et al. 2017). The coefficient is used to assess monotonic relationships between all possible combinations of unique indicator data pairs (Pradhan et al. 2017). To avoid wrong conclusions regarding synergies and tradeoffs (e.g., between poverty rate and electricity production from renewable energies) we provided all indicators with a positive or negative sign depending on whether the aim is to increase or to reduce them. For the analysis presented in Fig. 2 in "Results" section, we included only pairwise correlations with a $p$ value of less than 0.1 . While positive correlations $(\rho>0.6, p$ value $<0.1)$ indicate potential synergies (co-benefits), negative correlations $(\rho<-0.6$, $p$ value $<0.1$ ) indicate potential conflicts (trade-offs) between targets. Significant values between -0.6 and 0.6 are not classified as synergies or trade-offs so as to avoid overinterpreting the correlation coefficients as a measure of the strength of interaction between two variables (Hauke and Kossowski 2011; Pradhan et al. 2017). We could include 20 MONET 2030 indicators for priority targets for which we found at least four records for the period from 2000 to 2016. Indicators to which we could not assign a desired trend (e.g., sustainable timber use) were not included in this analysis.

When interpreting the results, it is important to keep in mind that indicators are primarily proxies describing a complex mechanism. This means that confounding factors or spurious correlations between indicators cannot be excluded. Accordingly, our results from this quantitative analysis provide a first rough overview of likely interactions between Swiss priority targets based on indicators available in MONET 2030.

\section{Results}

We present our results in the following order. First, we present the priority targets for Switzerland that we derived from stakeholders' assessments by applying an analytic hierarchy process. Second, we provide some general observations based on the cross-impact matrix of priority targets, including which targets have the greatest influence on other targets and which targets are highly dependent on progress towards other targets for their achievement. Third, we delve deeper into the analysis of interactions between targets and present the results from our network and prospective structural analyses. On this basis, we outline how action on implementing the 2030 Agenda can be guided or reprioritized. Finally, we compare the outcomes of these qualitative assessments with the results from our quantitative assessment of synergies and tradeoffs between SDG targets.

\section{Priority targets for Switzerland based on stakeholders' assessments}

As described in "Data and methods" section, we applied an analytic hierarchy process to identify priority targets for Switzerland based on an assessment of targets by stakeholders. The first 27 targets were selected for their political feasibility. An additional six targets were selected based on the second criterion of strong stakeholder group positions. Altogether, we selected 33 priority targets for Switzerland (Table 3). 
Table 3 Priority targets for Switzerland

\begin{tabular}{|c|c|c|}
\hline SDG target & Short description & $\begin{array}{l}\text { Selection criterion } \\
1=\text { political feasibility } \\
2=\text { strong stakeholder position }\end{array}$ \\
\hline 1.1 & Eradication of extreme poverty & 1 \\
\hline 1.2 & Reduction of multidimensional poverty & 1 \\
\hline 1.4 & Equal right to economic resources & 1 \\
\hline 1.a & Mobilization of resources to end poverty & 1 \\
\hline 2.1 & Ending hunger & 1 \\
\hline 2.3 & Increased agricultural productivity & 1 \\
\hline 2.4 & Sustainable and resilient agriculture & 1 \\
\hline 3.9 & Reduced air, water and soil pollution & 1 \\
\hline 4.7 & Knowledge for sustainable development & 1 \\
\hline 4.b & Scholarship for developing countries & 2 (science) \\
\hline 5.1 & Ending discrimination against woman and girls & 2 (social organizations) \\
\hline 6.1 & Safe drinking water & 2 (civil-society organizations) \\
\hline 7.1 & Modern energy services & 1 \\
\hline 7.2 & Increased share of renewables & 1 \\
\hline 7.3 & Doubled rate of improvement in energy efficiency & 1 \\
\hline 7.a & Enhanced international knowledge transfer & 1 \\
\hline 8.2 & Higher economic growth through diversification & 2 (private-sector organizations) \\
\hline 8.4 & Decoupling of economic growth from ecological resources & 1 \\
\hline 10.4 & Fiscal, wage and social protection policies for greater equality & 2 (public-sector organizations) \\
\hline 11.3 & Inclusive and sustainable urbanization & 1 \\
\hline 11.6 & Reduced environmental impact of cities & 1 \\
\hline 12.2 & Sustainable management and efficient use of resources & 1 \\
\hline 12.3 & Reduced global food waste & 1 \\
\hline 12.6 & Adoption of sustainable practices by companies & 1 \\
\hline 12.8 & Information and awareness for SD and lifestyle & 1 \\
\hline 13.1 & Strengthened resilience to climate-related hazards & 1 \\
\hline 13.2 & Integration of climate change measures into national policies & 1 \\
\hline 13.3 & Improved education & 1 \\
\hline 15.3 & Restoration of degraded land and soil & 2 (environmental organizations) \\
\hline 15.4 & Conservation of mountain ecosystems & 1 \\
\hline $15 . \mathrm{a}$ & Mobilization of resources for biodiversity & 1 \\
\hline 17.2 & Full implementation of ODA commitments & 1 \\
\hline 17.14 & Enhanced policy coherence for SD & 1 \\
\hline
\end{tabular}

\section{Synergies and trade-offs between Swiss stakeholders' priority targets: a qualitative assessment}

Following the analysis of stakeholders' priorities, we examined systemic interactions between targets by means of a cross-impact matrix (Fig. 2). The established matrix consists of 33 priority targets based on the analysis of stakeholders' priorities. The cells indicate the expert group's assessment of interactions based on the seven-point scale developed by Nilsson (2016).

The scoring of interactions between the determined priority targets was guided by the question: "If progress is made on target $x$ (rows) in Switzerland, how does this influence progress on target y (columns) in Switzerland and beyond"? The main focus was, thus, on interactions triggered by progress on the various targets, not by their full achievement. Moreover, the experts were asked to think more of direct effects than of possible indirect effects (as in: "If progress is made on target $x$, this might trigger $z$, which might influence progress on target $y . . . ")$. For instance, we assessed that an increased share of renewable energy (target 7.2) would have a reinforcing influence on progress towards reducing air, water, and soil pollution (target 3.9).

We determined a significant positive or negative influence in about $37 \%$ of the 1056 potential interactions. 
The cross-impact analysis for the targets prioritized by Swiss stakeholders further shows that negative interactions account for less than $2 \%$ of interactions (19 tradeoffs) and are by far outnumbered by positive interactions (368 co-benefits). The dominance of positive interactions between targets confirms observations made in other local contexts (Nilsson et al. 2018; Weitz et al. 2018) and at the global level (Pradhan et al. 2017; Breuer et al. 2019). However, there are important exceptions which need further attention. Notably, the targets causing trade-offs with other targets include targets 2.3 (double the agricultural productivity), 2.1 (end hunger), 7.1 (access modern energy services), 12.2 (sustainable management of natural resources), 13.2 (integrate climate change measure in national policies), 13.3 (education on climate change mitigation), and 15.4 (conservation of mountain ecosystems). In addition to the number of positive, neutral, and negative scorings, the cross-impact matrix offers further systemic insights. The row sums-the added-up scores of a target's impacts on all other priority targets-differ considerably between targets. A high row sum indicates that the given priority target has a high potential for synergies regarding progress on different priority targets. On this basis, we can assume that enhancing policy coherence for sustainable development (17.14), integrating climate change measures into national policies and strategies (13.2), restoring degraded land and soil (15.2), and encouraging companies to adopt sustainable practices and integrate sustainability information into their reporting cycles (12.6) would have the most benefits across several priority targets. Conversely, a low or negative row sum-as found for targets 2.3 (double agricultural productivity), 2.1 (nutritious food), 7.1 (access to energy services), and 3.9 (air, water and soil pollution)—indicates a high number of potential conflicts and the need for particular care when implementing actions to achieve the target within the Swiss context. A high column sum suggests that a target is greatly positively influenced by other targets. Target 13.1 (strengthen resilience and capacity to adapt to climate-related hazards) has the highest column sum, indicating that this is the most positively influenced target. Nevertheless, this target also receives a negative influence $(-2)$. The same is true for target 2.4 (food production/agriculture), which not only has the second-highest column sum (27) but also receives a negative influence $(-1)$. Progress on these highly influenced targets depends on developments in other targets and their independence or independent control over their own progress is low. These first insights based on the crossimpact matrix provide an overview of targets' net influence on other targets and whether they are strongly influenced by progress in other targets (dependence), but it does not provide enough information to guide priority-setting in terms of where to focus action. For that purpose, we need more nuanced information.

\section{Systemic multipliers and virtuous cycles}

As described in "Data and methods" section, we analyzed the positive and negative interconnectedness of targets within their network. This enabled us to determine whether they might act as potential systemic multipliers of co-benefit or trade-off effects.

Figure 3 provides insights into the systemic roles of the targets in the network. We can see that enhancing policy coherence for sustainable development (target 17.14) is the most positively influencing target (high activity ratio) and that target 2.1 is the most positively dependent one (low activity ratio). More importantly, however, the scatterplot shows that sustainable management and efficient use of resources (target 12.2), strengthened resilience to climaterelated hazards (target 13.1), and sustainable and resilience agriculture (target 2.4) — as buffers—as well as integration of climate change measures into national policies (target 13.2) and restoration of degraded land and soil (target 15.3) - as multipliers - are highly interconnected with the other targets in the network. According to this more nuanced interpretation of positive and negative roles, we find that increased agricultural productivity (target 2.3 ) and modern energy access (target 7.1) are positively dependent on the implementation of other targets, while they can negatively impact progress on other targets in the system. Moreover, we find that sustainable management and efficient use of resources (target 12.2) can be especially negatively impacted by the implementation of these targets. Finally, the plot visualizes the categorization of targets as buffers or multipliers of positive or negative effects (Fig. 3). Blue (or red) nodes to the right of the vertical line (activity ratio $<1$ ) represent systemic multipliers of positive (or negative) effects; those to the left of the vertical line (activity ratio $>1$ ) represent buffers.

While Fig. 3 shows that 2.3 and 7.1 could have significant negative effects on other targets and must hence be addressed with care, Fig. 4 further reveals that the following targets are likely to have positive effects across the whole system and thus on reaching the SDGs as a whole:

- Enhanced policy coherence for sustainable development (target 17.14)

- Restoration of degraded land and soil (target 15.3)

- Integration of climate change measures into national policies (target 13.2)

- Reduced global food waste (target 12.3)

- Adoption of sustainable practices by companies (target 12.6)

- Inclusive and sustainable urbanization (target 11.3) 


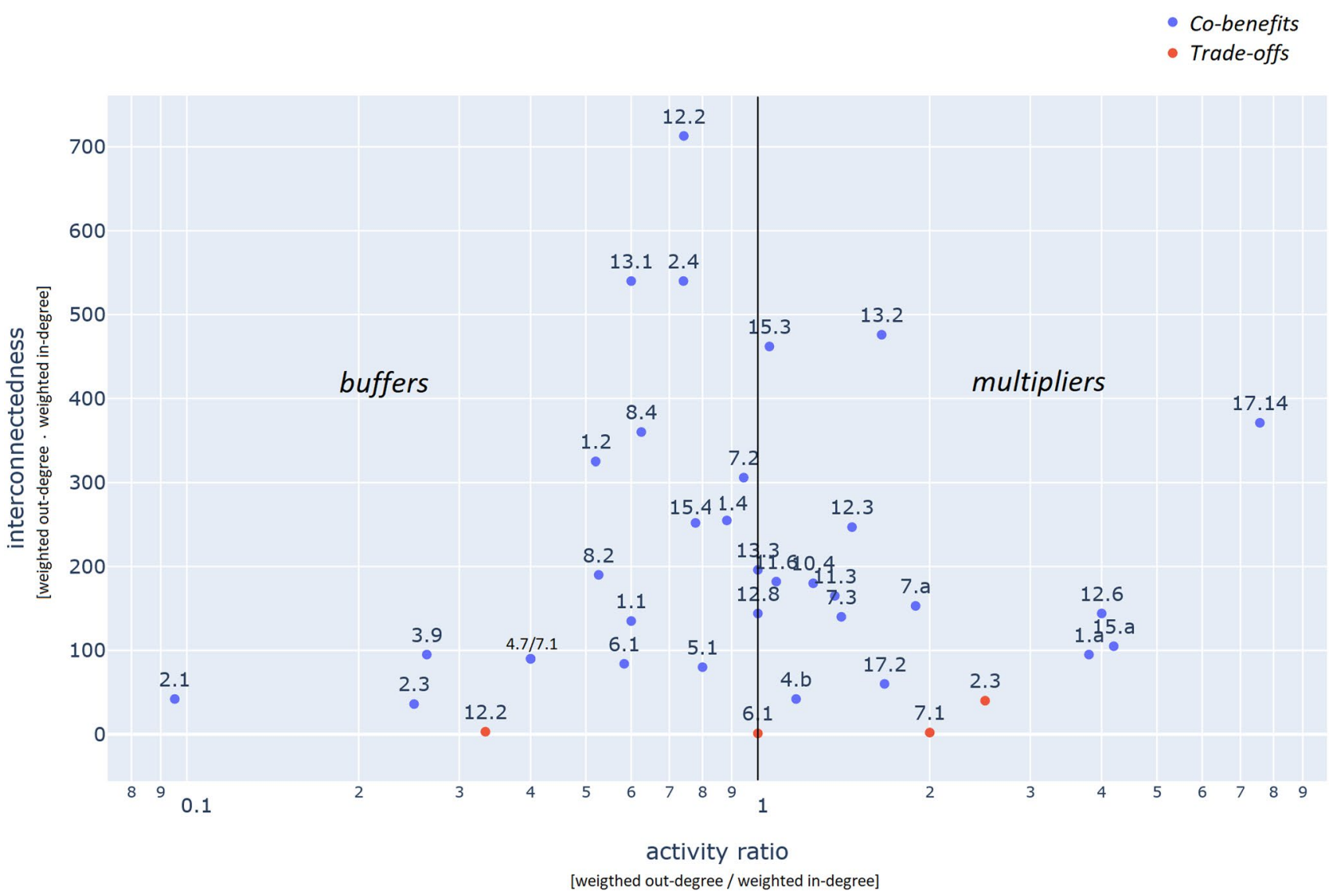

Fig. 3 Systemic role of the targets as buffers of multipliers for the better or the worse. Red nodes represent the trade-offs that a target is involved in, whereas blue nodes represent the synergies

- Reduced environmental impact of cities (target 11.6)

- Mobilization of resources to end poverty (target 1.a)

- Mobilization of resources for biodiversity (target 15.a)

Finally, well-connected buffers like sustainable management and efficient use of resources (target 12.2), strengthened resilience to climate-related hazards (target 13.1), sustainable and resilient agriculture (target 2.4), and decoupling of economic growth from ecological resources (target 8.4) have the potential to serve as outcomes that reveal the positive or negative trajectory towards sustainable development and achieving the SDGs as a whole in Switzerland.

The aim of our systemic approach is to move away from siloed monitoring and implementation, towards proactively utilizing the synergistic potential of the 2030 Agendawhich is the most promising way to effectively progress towards the SDGs as a whole. In line with this aim, our assessment of interactions among targets and our subsequent network analysis also enabled us to concretize in the Swiss context the ideas underlined in the GSDR. In particular, we found a number of virtuous cycles (Fig. 5) that point to potential transformation pathways and could thus serve as concrete entry points for implementing the 2030 Agenda in Switzerland (Independent Group of Scientists 2019).

Indeed, our extraction of all positive systemic loops of three targets revealed 22 virtuous cycles, which we condensed visually wherever possible (e.g., the loop from target 12.2 to target 12.3 to targets $8.4 / 15.3$ and back to target 12.2 indicates two loops, one involving target 8.4 and another involving target 15.3). Ordering these cycles according to their topical closeness to the six entry points for transformation proposed in the GSDR facilitates communication of the results.

To build human capabilities in Switzerland, one should consider the reinforcing effects of simultaneously tackling multidimensional poverty (1.1 and 1.2) and ensuring equal rights to economic resources (1.4) and social protection systems (10.4). On the way to more sustainable and just economies, committing to decoupling economic growth from ecological resources (8.4) and to sustainable and efficient use of resources (12.2) can support and at the same time benefit from efforts to tackle challenges like increasing the share of renewable energy (7.2) and reducing food waste (12.3). When working towards more sustainable food systems, 


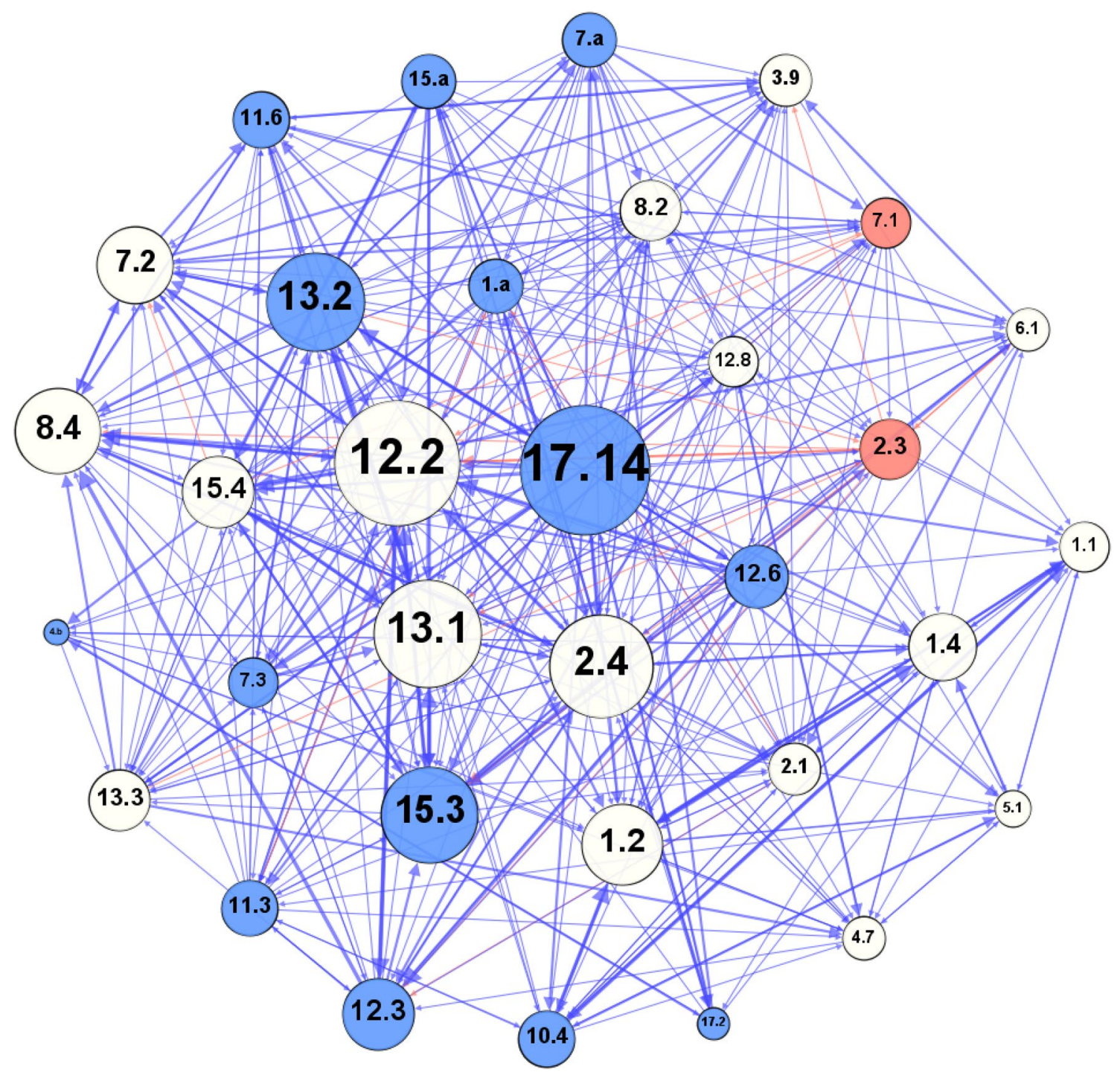

Fig. 4 Result of the analysis of systemic buffers (white) and multipliers of positive (blue) and negative (red) effects in the Swiss context. The node size is proportional to the weighted degree centrality and shows how connected a node is within the network

promotion of sustainable use of resources (12.2.) can support efforts to decouple economic growth from ecological resources (8.4), reduce food waste (12.3), and prevent land and soil degradation (15.3). All three of the above domains could contribute to encouraging sustainable agriculture (2.4), which in turn would further support sustainable resource use (12.2). Many identified pathways seem related to energy decarbonization. For instance, policy coherence for sustainable development (17.14) and sustainable resource use (12.2) can be directed to support renewables (7.2) and efficiency (7.3) in the energy sector, which in turn would strengthen national climate policies (13.2) — which could again reinforce policy coherence (17.14) and sustainable resource use (12.2). Increased shares of renewable energy (7.2) can also benefit urban and peri-urban development by helping to greatly reduce the environmental impact of cities (11.6); this in turn can contribute to a more sustainable economy (8.4), thereby supporting further energy decarbonization. Finally, protecting the environmental commons requires pathways based on the mutually reinforcing effects of preventing land and soil degradation (15.3), conserving mountain ecosystems (15.4), and strengthening resilience to climate-related hazards (13.1).

As the GSDR states, "[p]athways are integrated and context-specific combinations of levers to achieve transformational change towards sustainable development through the six entry-points" (Independent Group of Scientists 2019). Accordingly, embarking on pathways to sustainable development will imply combining the four levers (Governance, Economy and Finance, Individual 


$\begin{array}{llllll}\begin{array}{l}\text { Human well-being } \\ \text { and capabilities }\end{array} & \begin{array}{l}\text { Sustainable and } \\ \text { just economies }\end{array} & \begin{array}{l}\text { Sustainable food systems } \\ \text { and healthy nutrition }\end{array} & \begin{array}{l}\text { Energy decarbonization } \\ \text { with universal access }\end{array} & \begin{array}{l}\text { Urban and peri-urban } \\ \text { development }\end{array} & \begin{array}{l}\text { Global environmental } \\ \text { commons }\end{array}\end{array}$

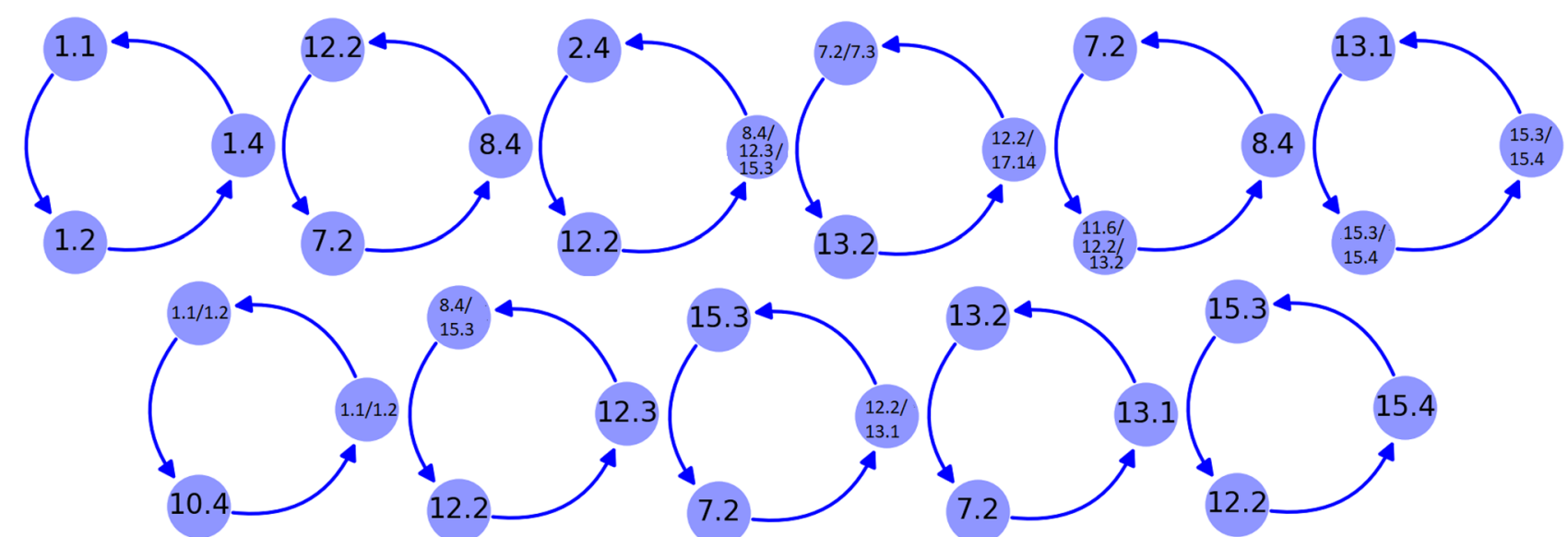

Fig. 5 Identification of virtuous cycles in the network, adapted from Pham-Truffert et al. (2020), condensed and ordered by the authors for their exemplification in the Swiss context of the six entry points for transformation proposed in the GSDR (Independent Group of Scientists, 2019)

and Collective Action, and Science and Technology) while involving relevant agents of change, creating solutions that make use of virtuous cycles, and formulating concrete action plans to implement them.

\section{Direct influence and dependence matrix}

Using prospective structural analysis as described in "Methods" section, we constructed an influence-dependence matrix (Fig. 6). The matrix is based on the calculated indegree centrality (influence), which indicates the general
Fig. 6 Direct influence-dependence matrix based on prospective structural analysis

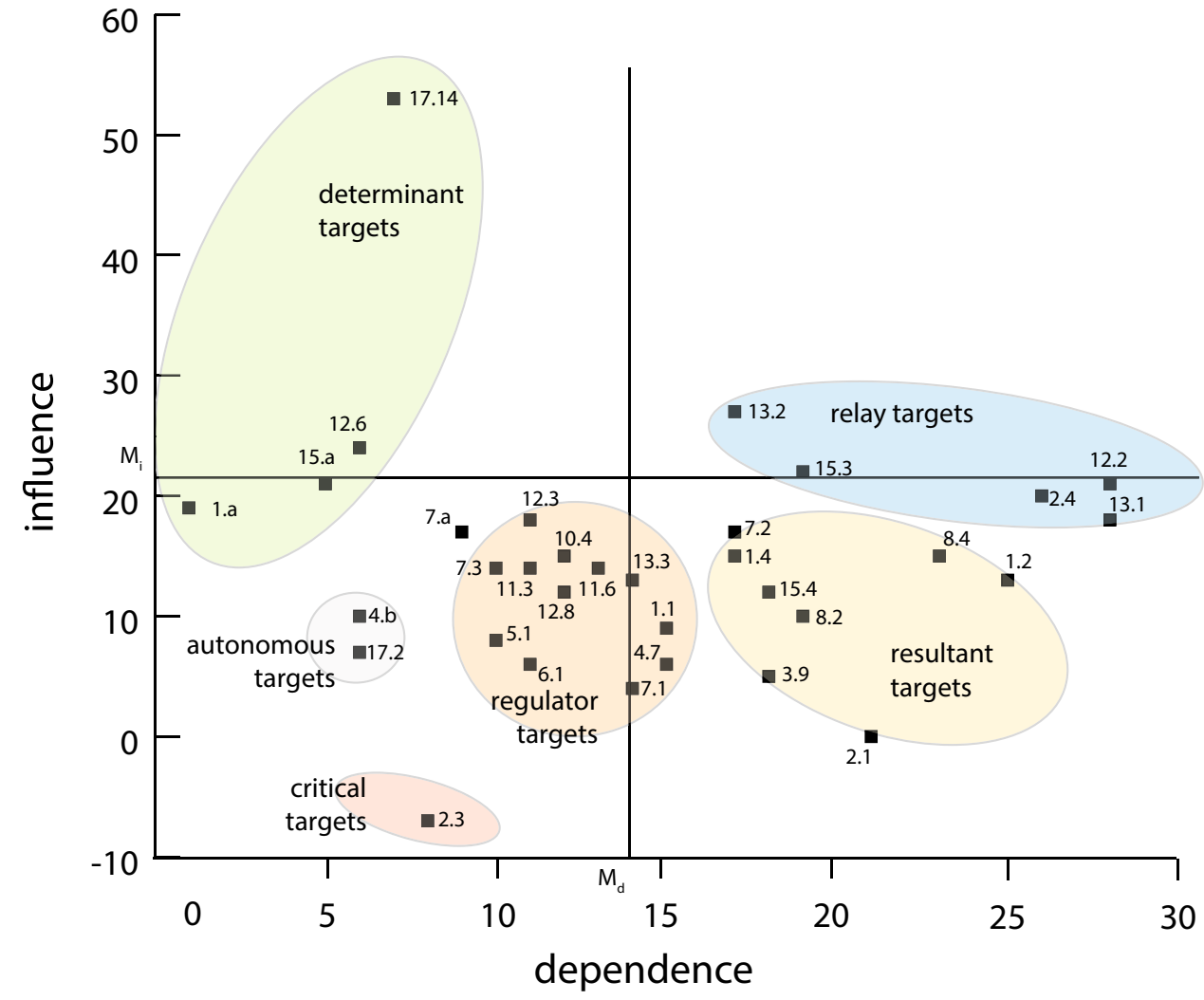


degree of influence a target has on all the other targets, and the out-degree centrality (dependence), which shows how dependent each target is on all the other targets.

Focusing on the implementation of the national 2030 Agenda, determinant targets and critical targets are highly influential and could play a significant role in achieving, or counteracting the achievement, of other targets. These targets should thus receive special attention and must be prioritized in the process of implementing the 2030 Agenda in Switzerland. Determinant targets in particular should be the primary focus, as progress on these could bring about progress on other targets (relay and resultant targets). In the case of Switzerland, this would mean prioritizing the following three actions: (1) recruiting financial resources for implementing programs and policies to end poverty in all its dimensions (target 1.a) and for protecting biodiversity and ecosystems (target 15.a); (2) encouraging large and transnational companies to adopt sustainable practices and integrate sustainability measures into their reporting cycles such that these entities can become leverage points for sustainability transformation (target 12.6); and (3) enhance policy coherence for sustainable development (target 17.14). Comparing the results from the prospective structural analysis (Fig. 6) with those from the identification of systemic multipliers (Fig. 3), one might argue that target 7.a could also be defined as a determinant target.

Targets $2.4,12.2,13.1,13.2$, and 15.3 can be described as relay targets. These targets have a strong influence on other targets and are themselves highly dependent on progress in other targets. It seems that the common denominator of these targets is "action on climate change", as target 2.4 (sustainable food production), 12.2 (sustainable management and efficient use of natural resources), 13.1 (strengthened resilience to climate-related hazards), 13.2 (integration of climate change measures into national policies) as well as 15.3 (restoration of degraded land and soil) have obvious links to climate debates. Regulator targets, despite their moderate dependence and influence, could act as levers for change-depending on how they are implemented. Implementation, in turn, depends on who has the power to dictate laws and regulations. For example, the influence of target 10.4 (adopt policies, especially fiscal, wage and social protection policies, and progressively achieve greater equality) on other targets will differ fundamentally, depending on whether we implement progressive ideas based on work by Piketty and Goldhammer (2014); Piketty (2019) and others or whether current opinion leaders succeed in protecting the status quo. Resultant targets are the most strongly influenced (dependent) ones. Their achievement depends on progress made towards determinant and relay targets. The combination of determinant and regulator targets is decisive for the achievement of resultant targets. For example, whether Switzerland succeeds in decoupling economic growth and use of natural resources (target 8.4) mainly depends on future consumption and production patterns, as well as how fast Switzerland achieves the energy transition. Autonomous targets have a low potential to generate change and advance the 2030 Agenda. In line with Godet (1991), we argue that these autonomous targets can be excluded from the analysis because they are not expected to influence the national implementation of the 2030 Agenda in Switzerland.

\section{Synergies and trade-offs between Swiss stakeholders' priority targets: a quantitative assessment}

While the above qualitative analysis of interlinkages between Swiss priority targets took an ex ante approach, asking how progress in target $x$ influences achievement of target $y$, a quantitative assessment based on time-series data for the years 2000-2016 takes an ex post perspective. Using nonparametric Spearman rank correlations, Fig. 7 presents the results obtained from this ex post perspective on interlinkages between Swiss priority targets.

Of the significant interactions between priority targets analyzed, 58\% were identified as potential synergies, $26 \%$ as potential trade-offs, and $16 \%$ as neutral. These findings are in line with other studies showing that in general, a solid majority of targets reinforce each other. Trade-offs are concentrated around targets 7.1, 8.4, and 10.4. This points towards the fact that these targets showed opposing trends between 2000 and 2016 compared to other priority targets. However, it is important to note that correlations might not represent causalities. They merely provide information about development over time, but not about whether two targets developed alongside each other or whether they followed opposing trends.

\section{Discussion}

In the following, we reflect on our results from a methodological point of view. We discuss the limitations of the tested approaches and methods and present ideas for their further development.

\section{Priority identification for national 2030 Agendas}

Our first step was to define priority targets and target areas for implementation of the 2030 Agenda in Switzerland based on the responses Swiss stakeholders gave in a national consultation process. This survey was designed to underpin and complement the government's own assessment with regard to Switzerland's progress towards the 2030 Agenda. It was also aimed at revealing where there was a need for additional action, particularly with a view to policy coherence, and 


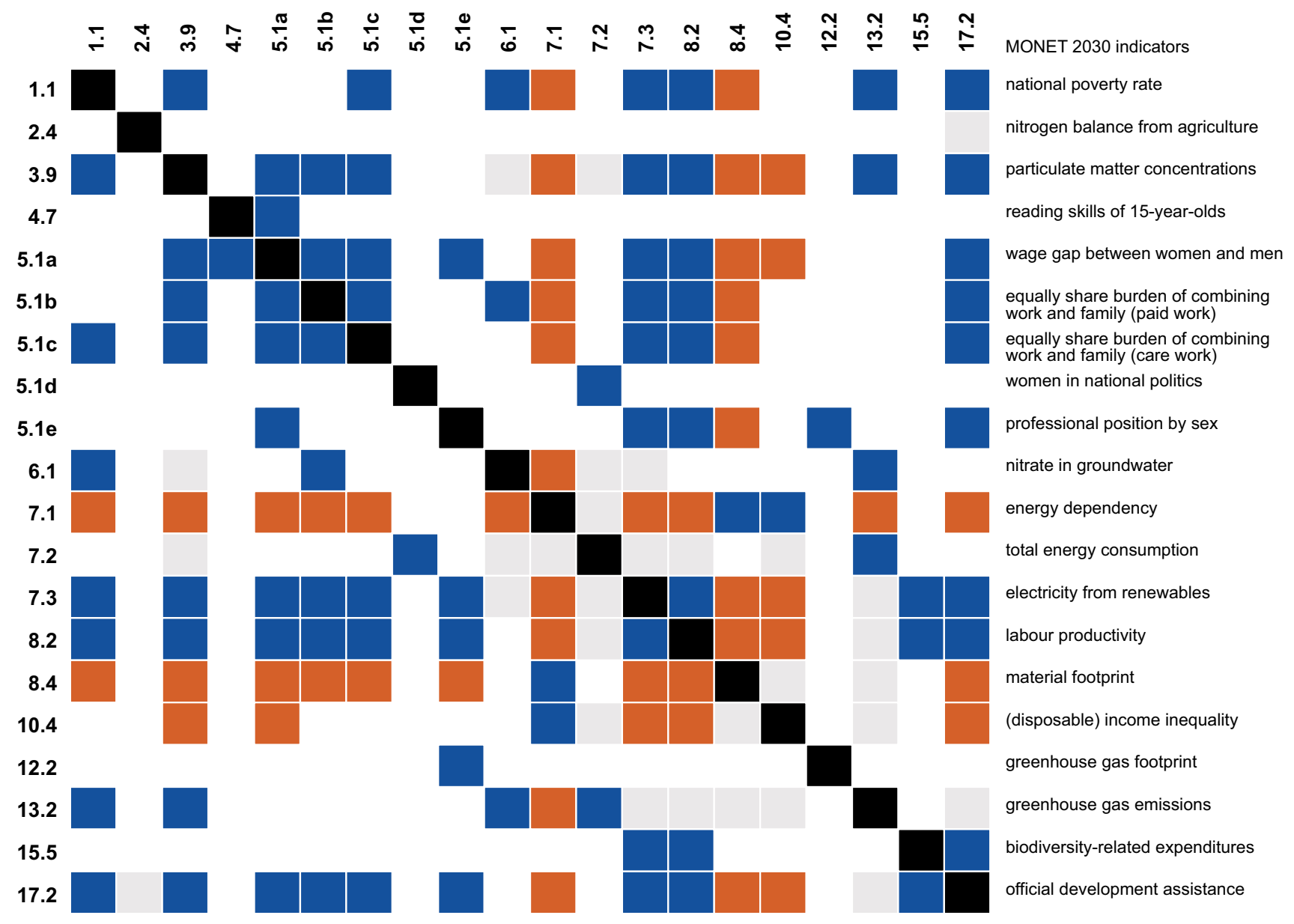

Fig.7 Quantitative assessment of interactions between priority targets for Switzerland (2000-2016). The color code represents different levels of significant correlations (dark red: $\leq-0.6$; gray: -0.6 to 0.6 ; dark blue: $\geq 0.6$ )

what opportunities could be harnessed. Such broad-based surveys among organizations from civil society, science, and the private sector constitute an indispensable instrument not only for defining priorities and opportunities for sustainable development, but also, and just as importantly, for triggering debates and for forming partnerships for implementation of the 2030 Agenda. Given that the survey was open to one respondent of any of non-governmental institution and the respondents were not selected based on a survey sampling method, its representativeness must be questioned regarding the weight of the six stakeholder groups. As shown in Table 1, social organizations, the private-sector, and civilsociety organizations clearly have an above-average number of respondents, while public-sector, environmental, and scientific organizations represent only $28 \%$ of the 167 respondents. Our analytic hierarchy process for identifying priority targets seems to be suited to counterbalance or at least mitigate the risk of overrepresentation of certain stakeholder groups. The process ensures that priority-setting takes into account the priorities across all stakeholder groups. In addition, the process also enabled targets of specific interest to a single stakeholder group (e.g., targets 15.3 on combating desertification, 4.b on scholarships for developing countries, and 5.1 on ending discrimination against women) to be included in the list of priority targets and thus ensured, at least to some extent, balanced representation of stakeholder groups with few respondents. A second bias in such a survey without a sampling approach concerns the respondents' interest in issues of sustainable development. Based on a qualitative review of the organizations to which the 167 anonymous respondents belonged, we must assume that they included a disproportionally high number of organizations who were interested or already engaged in sustainability issues; whereas, organizations with less interest in sustainable development tended not to participate. A further limitation of the survey concerns respondents' uncertainty regarding the system boundaries. The survey was designed to focus on Switzerland itself and did not systematically include concerns about spillover effects from Switzerland to other countries. The fact that respondents nonetheless 
frequently mentioned such concerns adds to the limits of this survey.

In order for such a stakeholder consultation to become a sound basis for interaction analysis and subsequent formulation of sustainable development strategies, due attention must be given to the representativeness of respondents. Particular care must be taken to including respondents who are not very concerned or informed about sustainable development. Given that the 2030 Agenda claims to be universal, the design of such surveys should systematically address spillover effects on other countries beyond obvious policy fields such as climate change, trade and investment, taxes and finances, or development cooperation.

\section{Appraising systemic interactions between priority targets}

Given the general orientation of this article, in the following paragraphs, we present some methodological reflections on assessing interactions using the qualitative approach of Nilsson et al. (2016) and the indicator-based quantitative approach of Pradhan et al. (2017).

Since Nilsson et al. (2016) first proposed it, the crossimpact matrix has received considerable recognition and has been applied and further developed in many different contexts (International Council for Science 2017; Nilsson et al. 2018; Weitz et al. 2018; for an overview, see Tosun and Leininger 2017; Breuer et al. 2019). It has proved to be an intuitive, fairly simple, and easily replicable framework for mapping and analyzing the relationships between SDGs and SDG targets, and has been used to structure knowledge accumulation and policy learning when setting priorities for implementation of the SDGs and efforts towards PCSD (OECD 2018; Allen et al. 2019). Methodologically, it helps to overcome the dichotomy or simplified "binary view" of synergies versus trade-offs by qualifying the relationships between targets (Nilsson et al. 2018; Weitz et al. 2018). Nevertheless, Nilsson et al. pointed out methodological limitations early on, and others have elaborated further on this. In particular, they have shown that time horizon, geographical context, governance issues, technology, and directionality fundamentally affect the scoring results (Nilsson et al. 2016; Weitz et al. 2018; Breuer et al. 2019). Furthermore, system interactions are infinite, and target selection as well as expert judgements strongly depend on perceptions and are therefore no less normative than the SDG framework as a whole. To account for the normative nature of the SDG framework, scholars have emphasized the importance of joint scoring exercises among scientists, civilsociety representatives, and policymakers (Lubchenco et al. 2015; Breuer et al. 2019) as part of an "analytic-deliberative hybrid process [...] entailing both formal evidence and hard data, expert judgment, and stakeholder-driven deliberative data generation" (Nilsson et al. 2018).

The qualitative appraisal of SDG target interactions as applied in this study goes in the direction of such a hybrid process. Unlike in other applications, where a subset of important targets for the given country was chosen on the basis of expert judgement (Weitz et al. 2018), our cross-impact matrix builds on an assessment of priority targets by Swiss stakeholders. During our scoring exercise, we learned that the harmonization of the individual experts' scorings raises countless discussions around the above methodological challenges. This occurred particularly when seeking a consensus regarding the time horizon and the system boundaries for spillover effects, as well as when dealing with first- and second-order effects. The availability of a detailed country-specific translation of the 2030 Agenda based on societal negotiation (Nilsson et al. 2018), with transparently exposed supporting facts and knowledge (Pham-Truffert et al. 2019), would help to limit uncertainties and reduce incoherencies in such qualitative appraisals.

Compared to our qualitative appraisal of interlinkages, the quantitative approach of Pradhan et al. (2017) has considerably more limitations. As a result, its national-level application does not make sense, be it in Switzerland or in most other countries. First of all, the correlation analysis shows only associations between indicators, not causalities, and it only accounts for first-order effects (Breuer et al. 2019). Second, in most countries, indicator data will hardly be available in sufficient quantity and quality, even for a relatively small set of priority targets, leading to arbitrary results. The Swiss MONET 2030 monitoring system we used in this study currently contains only 85 indicators, and for a number of them, there were no sufficiently long time series. In our study, we found only 20 indicators for the 33 identified priority targets that meet the requirement of at least four records for the period from 2000 to 2016. Third, indicator systems are generally developed by statisticians in consultation with the respective administrative units and cannot normally rely on country-specific translations of the 2030 Agenda. Lastly, like many other national indicator systems, the MONET 2030 system and its indicators were not designed for analyzing the interactions between different sustainable development targets. This is not least reflected by the fact that they include both absolute and relative figures, which is unsuitable for a quantitative analysis of interactions. Given the above considerations, the different further analytical steps in this study built on the results of the qualitative assessment.

\section{Prioritizing policy action on implementing the $\mathbf{2 0 3 0}$ Agenda at the national level}

Despite the indivisible nature of the 2030 Agenda and its SDGs and targets, we have split them up into tangible 
entities using cross-impact matrices and network analysis. As a result, we ended up with 33 targets and 1056 interactions. Although most interactions were classified as positive, meaning that targets mutually reinforce each other in a desired way, there are also negative interactions, where targets undermine or contradict each other. The results and information contained in the cross-impact matrix could, thus, potentially provide useful starting points for national governments, e.g., to better understand the interconnectedness of targets and identify those that present particular challenges (target 2.4 for Switzerland) and opportunities in their national contexts.

However, the big question of where to begin has yet to be solved. As a first step to answering it, we used prospective structural analysis to establish an order of priority policy action on implementing the 2030 Agenda at the national level. We think this is a promising avenue to understanding where to begin with the national implementation of the 2030 Agenda. Nevertheless, given the subjective nature of judging the interactions between selected priority targets-a task performed by five sustainability experts with profound knowledge of the Swiss context-we propose to complement our chosen approach with a cross-check by the consulted stakeholders, leading to a "qualified" priority order for policy action. The proposed cross-check might be performed by means of a conventional Delphi process (Linestone and Turoff 1975). Such a procedure would ensure that the generated results based on expert judgment of interactions between priority targets and network analysis is meaningful and can be translated into practical value for policymakers. Furthermore, setting up a Delphi round with results generated e.g. in Fig. 6 could lead to a re-evaluation of interactions between targets. Finally, based on such a qualified priority order for policy action and the derivation of "virtuous cycles", we could think of organizing a co-design process together with stakeholders to concretize these pathways and entry points for transformation in the Swiss context.

\section{Conclusions for designing national 2030 Agenda strategies}

The purpose of the present study is to contribute to the methodological conceptualization of the implementation of the 2030 Agenda at the national level. To this end, we tested, enhanced, and discussed existing approaches for assessing interactions among the 2030 Agenda's SDG targets and for analyzing their systemic relevance. Based on our results, and taking into account the road map of Breuer et al. (2019), we propose a process of creating knowledge for implementation of the SDGs. The proposed process consists of eight steps involving both academic and non-academic stakeholders. We have tested steps 1-4 empirically, using Switzerland as an example; whereas, steps 5-8 reflect current debates on the implementation of the 2030 Agenda.

1. Translation of the 2030 Agenda to national contexts

The 2030 Agenda depends to a large degree on national progress towards sustainable development. One of the Agenda's guiding principles is that of Common but Differentiated Responsibility (CBDR), which was coined at the Rio 1992 World Summit. This principle acknowledges that countries' responsibilities must be differentiated because of disparities in capabilities (Williams and Montes 2016) as well as contextual differences. Against this background, translation of the 2030 Agenda to national contexts constitutes a prerequisite for defining, implementing, and monitoring progress on any national sustainability strategies. Researchers, in partnership with civil society, business, and policy, can contribute to translating the SDGs and their targets to specific contexts by providing knowledge (e.g., on social-ecological system dynamics) and engaging in dialogs organized by governments or civil-society organizations (Jacobi et al. 2020).

2. Identification of priority targets

Priority setting is indispensable, as the 2030 Agenda's 169 targets cannot be implemented as whole and the importance and urgency of the various targets vary widely between countries. Given the Agenda's normative character, it is essential to get an understanding of the perceived problem situation (Breuer et al. 2019). To this end, it is important to obtain substantiated knowledge about different stakeholder groups' perspectives on both the current condition and the targeted state of development. Such a survey must be based on a translation of the 2030 Agenda to the respective national context. Further, the survey design must meet scientific standards and ensure an adequate representation of all stakeholder groups. As shown in this study, there are methods that account for priority across all respondents while ensuring that the specific interests of individual stakeholder groups' are also represented.

3. Systemic assessment of SDG target interactions

Scientists widely agree that progress on the nationallevel implementation of the 2030 Agenda depends on our understanding and addressing interactions between its targets. They underline that implementation of the 2030 Agenda requires a systemic approach that focuses on interlinkages between goals and targets (Nilsson et al. 2016). The challenge lies in identifying and countering inherent conflicts (trade-offs) while harnessing and building on potential synergies (co-benefits) between the 169 targets. Although there are a growing number of instruments for analyzing SDG target interactions, they still present fundamental conceptual, practical, 
and procedural challenges in practice. In this study, we showed that the cross-matrix approach applying a sevenpoint scale (Nilsson et al. 2016) is better suited to the national level than indicator-based assessment methods (see Pradhan et al. 2017). This applies in particular if the selection of targets to be assessed is based on multistakeholder priority target assessment rather than simply relying on expert assessments (see Krohn et al. 2017). This means that joint knowledge generation among researchers, civil-society representatives, and policymakers (Lubchenco et al. 2015; Breuer et al. 2019) in support of an "analytic-deliberative hybrid process" (Nilsson et al. 2018) is to be preferred.

4. Identification of priority transformative pathways

To meet the indivisible nature of the 2030 Agenda, a systemic approach that simultaneously addresses multiple targets-rather than focusing on siloed subsets of targets-is most promising. The Global Sustainable Development Report 2019 (Independent Group of Scientists 2019) underlines that entry points for transformative pathways should address the underlying systems. Analytical knowledge on trade-offs and co-benefits among targets is key to defining context-specific transformative pathways. On the one hand, the analysis of targets' systemic roles can provide important guidance for efficient policy prioritization with regard to SDG governance. Building on the results of the interaction assessment, we showed from a systemic perspective how it is possible to identify the multipliers of positive or negative effects among priority targets. On the other hand, the derivation of strongly directed reinforcing loops ("virtuous circles") makes it possible to identify concrete transformative pathways and entry points for implementing the SDGs in Switzerland. As a further basis for defining national 2030 Agenda implementation strategies, we demonstrated the potential of prospective structural analysis (PSA) to support priority-setting with regard to coherent policy action towards the SDGs.

5. Accounting for winners and losers

Due attention must be given to the overarching notion of leaving no one behind while designing and implementing the 2030 Agenda. It is, thus, key to reflect on ethical questions when formulating national sustainability strategies. In concrete terms, the national 2030 Agendas must take into account how costs and benefits of planned interventions will be distributed across society, who will be the winners and losers, and how decisions are taken (Schneider et al. 2019). This requires the creation of target knowledge (see ProClim 1997) on the norms and values of a more desirable development (Kueffer et al. 2019), along with a transdisciplinary analysis of stakeholders' sustainability values, worldviews, and power relations (Schneider et al. 2019).
6. Policy coherence for sustainable development

Of paramount importance to progress on national implementation of the 2030 Agenda is policy coherence. Ensuring it takes an integrated effort across different policy areas to minimize trade-offs. This requires overcoming sectoral policy boundaries and better aligning rules and regulations, with the aim of simultaneously achieving multiple goals that are interlinked across sectors. Moreover, integrated policymaking, institutional and legal reforms, as well as skills are needed to support sustainable development (Breuer et al. 2019). To draft consistent sustainable development strategies, we will need knowledge and integrated approaches that take into account systemic interactions and causal relationships between SDG targets and policies (Independent Group of Scientists 2019).

7. Mapping of stakeholders

Novel collaborations and collective engagement among the scientific community, civil society, the public sector, and businesses are a prerequisite for triggering transformations towards sustainable development at the national level. National 2030 Agenda strategies should, therefore, reflect on what stakeholders might act as agents of change, and how they can work the levers proposed in the GSDR (Governance, Economy and finance, Individual and collective action, and Science and technology) to achieve the identified transformation pathways (Independent Group of Scientist 2019). Knowledge about stakeholders, their interests, and their power to influence implementation of the SDGs must be generated. This kind of mapping would have to address stakeholders' capacities, their needs, and not least their willingness for engagement (UNESCAP 2018).

8. Monitoring and evaluation of transformative pathways

National indicator systems not only need to be expanded to include more and better indicators, but also they must be adjusted such that they are suitable for monitoring transformative pathways and supporting reflection and social learning followed by adapted new action (Independent Group of Scientists 2019). Further, monitoring and evaluation systems must be established to enable reviewing and steering in the sphere of policy coherence for sustainable development (see OECD 2016; Collste et al. 2017; Nilsson and Weitz 2019). This should become a constituent part of future-oriented monitoring systems. In this respect, we must urgently develop new monitoring approaches capable of addressing multiple goals and related policies. An interesting approach for adopting an interaction perspective are relational indicators as applied in Germany's "Annual Well-Being Report" (Jahreswohlstandsbericht) (Zieschank and Diefenbacher 2019). Such indicators depict the gap between the current status and a desired target 
condition and constitute an ideal basis for communication and negotiations. They could also be an ideal vehicle for engaging and enlisting the commitment of scientists, policymakers, and civil society to work together to continuously review and guide countries' efforts towards achieving the 2030 Agenda.

Funding Open access funding provided by University of Bern.

Open Access This article is licensed under a Creative Commons Attribution 4.0 International License, which permits use, sharing, adaptation, distribution and reproduction in any medium or format, as long as you give appropriate credit to the original author(s) and the source, provide a link to the Creative Commons licence, and indicate if changes were made. The images or other third party material in this article are included in the article's Creative Commons licence, unless indicated otherwise in a credit line to the material. If material is not included in the article's Creative Commons licence and your intended use is not permitted by statutory regulation or exceeds the permitted use, you will need to obtain permission directly from the copyright holder. To view a copy of this licence, visit http://creativecommons.org/licenses/by/4.0/.

\section{References}

Allen C, Metternicht G, Wiedmann T (2019) Prioritising SDG targets: assessing baselines, gaps and interlinkages. Sustain Sci 14:421438. https://doi.org/10.1007/s11625-018-0596-8

Breu T, Bergöö M, Buergi Bonanomi E, Fässler M (2017) Switzerland and the Agenda 2030

Breuer A, Janetschek H, Malerba D (2019) Translating sustainable development goal (SDG) interdependencies into policy advice. Sustainability 11:2092. https://doi.org/10.3390/su11072092

Collste D, Pedercini M, Cornell SE (2017) Policy coherence to achieve the SDGs: using integrated simulation models to assess effective policies. Sustain Sci 12:921-931. https://doi.org/10.1007/s1162 5-017-0457-x

Dohlman E (2016) The importance of a policy coherence lens for implementing the Sustainable Development Goals. 20:37-40. https://doi.org/10.1787/9789264264687-7-en

Godet M (1991) From anticipation to action: a handbook of strategic prospective. UNESCO Publishing, Paris

Godet M (2007) Manuel de prospective stratégique-Tome 2-3ème edition-L'Art et la méthode. DUNOD, Paris

Gordon TJ, Hayward H (1968) Initial experiments with the cross impact matrix method of forecasting. Futures 1:100-116. https:// doi.org/10.1016/S0016-3287(68)80003-5

Griggs D, Stafford Smith M, Rockström J et al (2014) An integrated framework for sustainable development goals. Ecol Soc. https:// doi.org/10.5751/ES-07082-190449

Hauke J, Kossowski T (2011) Comparison of values of Pearson's and Spearman's correlation coefficients on the same sets of data. Quest Geogr 30:87-93. https://doi.org/10.2478/v10117-011-0021-1

Independent Group of Scientists (2019) Global sustainable development report 2019: the future is now-science for achieving sustainable development. United Nations, New York

International Council for Science (ICSU) (2017) A guide to SDG interactions: from science to implementation. International Council for Science (ICSU)
Jacobi J, Llanque A, Bieri S et al (2020) Utilization of research knowledge in sustainable development pathways: insights from a transdisciplinary research-for-development programme. Environ Sci Policy 103:21-29. https://doi.org/10.1016/j.envsci.2019.10.003

Krohn W, Grunwald A, Ukowitz M (2017) Transdisziplinäre Forschung revisited : erkenntnisinteresse, Forschungsgegenstände, Wissensform und Methodologie. GAIA Ecol Perspect Sci Soc 26:341347. https://doi.org/10.14512/gaia.26.4.11

Kroll C, Warchold A, Pradhan P (2019) Sustainable development goals (SDGs): are we successful in turning trade-offs into synergies? Palgrave Commun 5:140. https://doi.org/10.1057/s4159 9-019-0335-5

Kueffer C, Schneider F, Wiesmann U (2019) Addressing sustainability challenges with a broader concept of systems, target, and transformation knowledge. https://www.ingentaconnect.com/content/ oekom/gaia/2019/00000028/00000004/art00013\#. Accessed 19 Mar 2020

Le Blanc D (2015) Towards integration at last? The sustainable development goals as a network of targets. Sust Dev 23:176-187. https ://doi.org/10.1002/sd.1582

Linstone HA, Turoff M (1975) The Delphi method: techniques and applications, 1st edn. Addison-Wesley Educational Publishers Inc, Reading

Lubchenco J, Barner AK, Cerny-Chipman EB, Reimer JN (2015) Sustainability rooted in science. Nat Geosci 8:741-745. https://doi. org/10.1038/ngeo2552

Messerli P (2000) Use of sensitivity analysis to evaluate key factors for improving slash-and-burn cultivation systems on the eastern escarpment of Madagascar. Mt Res Dev 20:32-41. https://doi. org/10.1659/0276-4741(2000)020[0032:UOSATE]2.0.CO;2

Messerli P, Kim EM, Lutz W et al (2019) Expansion of sustainability science needed for the SDGs. Nat Sustain 2:892-894. https://doi. org/10.1038/s41893-019-0394-Z

Nilsson M, Chisholm E, Griggs D et al (2018) Mapping interactions between the sustainable development goals: lessons learned and ways forward. Sustain Sci 13:1489-1503. https://doi.org/10.1007/ s11625-018-0604-Z

Nilsson M, Griggs D, Visbeck M (2016) Map the interactions between sustainable development goals. Nat News 534:320. https://doi. org/10.1038/534320a

Nilsson M, Weitz N (2019) Governing trade-offs and building coherence in policy-making for the 2030 agenda. Polit Govern 7:254263. https://doi.org/10.17645/pag.v7i4.2229

OECD (2016) Better policies for sustainable development 2016: a new framework for policy coherence. OECD, Paris

OECD (2018) Policy coherence for sustainable development 2018: towards sustainable and resilient societies. OECD Publishing, Paris

Oliveira A, Calili R, Almeida MF, Sousa M (2019) A systemic and contextual framework to define a country's 2030 agenda from a foresight perspective. Sustainability 11:6360. https://doi.org/10.3390/ su11226360

Pham-Truffert M, Rueff H, Messerli P (2019) Knowledge for sustainable development: interactive repository of SDG interactions. In: CDE datablog. https://datablog.cde.unibe.ch/index .php/2019/08/29/sdg-interactions/. Accessed 30 Sep 2019

Pham-Truffert M, Metz F, Fischer M et al (2020) Interactions among sustainable development goals: knowledge for identifying multipliers and virtuous cycles. Sustain Dev 2020:1-15. https://doi. org/10.1002/sd.2073

Piketty T (2019) Capital et idéologie. Seuil, Paris

Piketty T, Goldhammer A (2014) Capital in the twenty-first century. The Belknap Press of Harvard University Press, Cambridge Massachusetts 
Pradhan P, Costa L, Rybski D et al (2017) A systematic study of sustainable development goal (SDG) interactions. Earth's Future 5:1169-1179. https://doi.org/10.1002/2017EF000632

ProClim (1997) Research on sustainability and global change-visions in science policy by Swiss Researchers. ProClim, Bern

Schneider F, Kläy A, Zimmermann AB et al (2019) How can science support the 2030 Agenda for Sustainable Development? Four tasks to tackle the normative dimension of sustainability. Sustain Sci 14:1593-1604. https://doi.org/10.1007/s11625-019-00675-y

Stafford-Smith M, Griggs D, Gaffney O et al (2017) Integration: the key to implementing the Sustainable Development Goals. Sustain Sci 12:911-919. https://doi.org/10.1007/s11625-016-0383-3

Swiss Civil Society Platform Agenda 2030 (2018) How sustainable is Switzerland? Implementing the 2030 Agenda from a civil society perspective. Plattform Agenda 2030, Bern, Switzerland

Swiss Confederation (2018) Switzerland implements the 2030 agenda for sustainable development. Switzerland's Country Report 2018. Bern, Switzerland

Swiss Federal Council (2016) Sustainable development strategy 20162019. Swiss Federal Council, Bern

Tosun J, Leininger J (2017) Governing the interlinkages between the sustainable development goals: approaches to attain policy integration. Glob Challenges 1:1700036. https://doi.org/10.1002/ gch2.201700036

UN DESA (2019) Handbook for the preparation of voluntary national reviews. United Nations

UNDP (2017) Rapid integrated assessment (RIA) tool to facilitate mainstreaming of SDGs into national and local plans. New York

UNESCAP (2018) Effective stakeholder engagement for the 2030 agenda
United Nations (2015) Transforming our world: the 2030 agenda for sustainable development

United Nations (2018) Voluntary national review reports-what do they report? New York

Weitz N, Carlsen H, Nilsson M, Skånberg K (2018) Towards systemic and contextual priority setting for implementing the 2030 Agenda. Sustain Sci 13:531-548. https://doi.org/10.1007/s1162 5-017-0470-0

Williams M, Montes MF (2016) Common but differentiated responsibilities: which way forward? Development 59:114-120. https:// doi.org/10.1057/s41301-017-0097-6

Zeigermann U (2018) Governing sustainable development through 'policy coherence'? The production and circulation of knowledge in the EU and the OECD. Eur J Sustain Dev 7:133-133. https:// doi.org/10.14207/ejsd.2018.v7n1p133

Zhou X, Moinuddin M (2017) Sustainable development goals interlinkages and network analysis: a practical tool for SDG integration and policy coherence. Institute for Global Environmental Strategies (IGES)

Zieschank R, Diefenbacher H (2019) Der Status Quo wird zum Risiko. Jahreswohlstandsbericht 2019. Fraktion Bündnis 90/Die Grünen im Deutschen Bundestag, Berlin

Publisher's Note Springer Nature remains neutral with regard to jurisdictional claims in published maps and institutional affiliations. 